

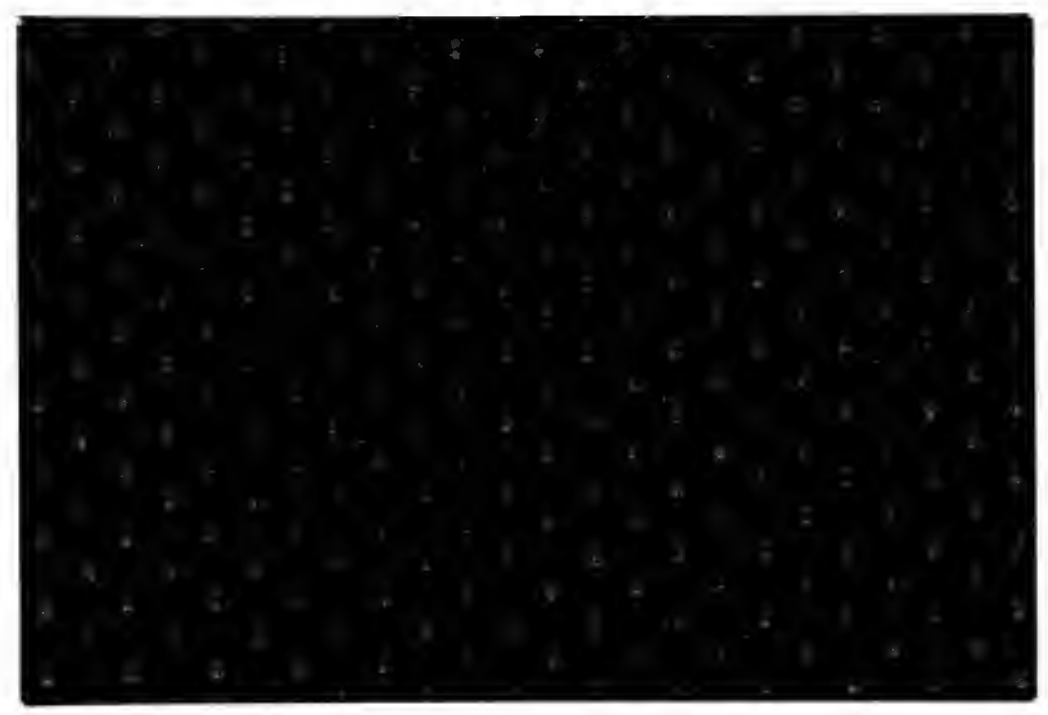




\title{
Almost Linear Upper Bounds \\ on the Length of General \\ Davenport-Schinzel Sequences
}

by

Micha Sharir

Technical Report No. 199

Robotics Report No. 61

February, 1985

\author{
New York University \\ Dept. of Computer Science \\ Courant Institute of Mathematical Sciences \\ 251 Mercer Street \\ New York, New York 10012
}

Work on this paper has been supported in part by a grant from the U.S.-Israeli Binational Science Foundation.

This paper is also Computer Science Technical Report No. 29/85 of The Eskenasy Institute of Computer Sciences, School of Mathematical Sciences, Tel-Aviv University, Tel-Aviv 69978, Israel. 



\title{
Almost Linear Upper Bounds on the Length of General Davenport-Schinzel Sequences
}

\author{
Micha Sharir \\ School of Mathematical Sciences \\ Tel-Aviv University \\ Tel-Aviv 69978, Israel
}

\begin{abstract}
Davenport-Schinzel sequences are sequences that do not contain forbidden subsequences of alternating symbols. They arise in the computation of the envelope of a set of functions. We obtain almost linear upper bounds on the length $\lambda_{s}(n)$ of Davenport-Schinzel sequences composed of $n$ symbols in which no alternating subsequence is of length greater than $s+1$. These bounds are of the form $O\left(n \alpha(n)^{\delta\left(\alpha(n)^{s-3}\right)}\right)$, and they generalize and extend the tight bound $\Theta(n \alpha(n))$ obtained by Hart and Sharir for the special case $s=3(\alpha(n)$ is the functional inverse of Ackermann's function), and also improve the upper bound $O\left(n \log ^{*} n\right)$ due to Szemeredi.
\end{abstract}

\section{Introduction}

Consider the following combinatorial problem (most of this introductory material also appears in [HS]): Let $n, s$ be positive integers. A sequence $U=\left(u_{1}, \ldots, u_{m}\right)$ of integers is an $(n, s)$ Davenport-Schinzel sequence (a $\operatorname{DS}(n, s)$ sequence for short), if it satisfies the following conditions:

(i) $1 \leq u_{l} \leq n$ for each $i$.

(ii) For each $i<m$ we have $u_{i} \neq u_{i+1}$.

(iii) There do not exist $s+2$ indices $1 \leq i_{1}<i_{2}<\cdots<i_{s+2} \leq m$ such that $u_{i_{1}}=u_{i_{3}}=u_{i_{5}}=\cdots=a, u_{i_{2}}=u_{i_{4}}=u_{i_{6}}=\cdots=b$, and $a \neq b$.

We will write $|U|=m$ for the length of the sequence $U$.

Define

$$
\lambda_{s}(n)=\max \{|U|: U \text { is a } D S(n, s) \text { sequence }\} .
$$

The goal of this paper is to establish almost linear upper bounds on $\lambda_{s}(n)$.

The problem of estimating $\lambda_{s}(n)$ has originally been posed by Davenport and Schinzel [DS]. Their interest in it arose from its connection to the

Work on this paper has been supported in part by a grant from the U.S.-Israeli Binational Science Foundation. 
analysis of solutions of linear differential equations. Recently, Atallah [At] has raised it again independently, because of its significance for problems in dynamic computational geometry. These two applications are quite similar, and can be briefly described as follows. Let $f_{1}, \ldots, f_{n}$ be $n$ real-valued continuous functions defined on a common interval $I$. Suppose that for each $i \neq j$ the functions $f_{t}$ and $f_{j}$ intersect in at most $s$ points (e.g., this is the case for polynomials of fixed degree, or Chebycheff systems, and so on). Let $g(x)=\min \left\{f_{i}(x): i=1, \ldots, n\right\}$, for $x \in I$, be the lower envelope (i.e. the pointwise minimum) of the $f_{i}$ 's, and let $m$ be the smallest number of subintervals $I_{1}, \ldots, I_{m}$ of $I$ such that for each $k$ there exists an index $i_{k}$ with $g(x)=f_{l_{k}}(x)$ for all $x \in I_{k}$. In other words, $m$ is the number of connected portions of the graphs of the $f_{l}$ 's which constitute the graph of $g$. Assuming that $I_{1}, \ldots, I_{m}$ are arranged in this order from left to right, put

$$
U\left(f_{1}, \ldots, f_{n}\right)=\left(i_{1}, \ldots, i_{m}\right) \text {. }
$$

It is now easily seen that $U\left(f_{1}, \ldots, f_{n}\right)$ is a $D S(n, s)$ sequence. Moreover, it is known (sce [At]) that for any $D S(n, s)$ sequence $U$ one can construct a collection $f_{1}, \ldots, f_{n}$ of such functions for which $U\left(f_{1}, \ldots, f_{n}\right)=U$. Therefore the largest possivic value of $m$ is precisely $\lambda_{s}(n)$.

Thus, in this setting, Davenport-Schinzel sequences are strongly related to the problem of computing the (lower) envelope of a set of functions which intersect each other in pairs in at most some fixed number of points. This problem has many applications in computational geometry and related areas, many of which are given in [At] and in [HS].

The problem of estimating $\lambda_{s}(n)$ has been studied in several papers [DS], [Da], [RS], [Sz], [At], [HS]. It is known (and easy to prove) that $\lambda_{1}(n)=n$ and $\lambda_{2}(n)=2 n-1$. Hart and Sharir [HS] have shown that $\lambda_{3}(n)=\Theta(n \alpha(n))$ by establishing an equivalence between $D S(n, 3)$ sequences and certain sequences of generalized path compressions on trees, and then by analyzing these path compression schemes. Here $\alpha(n)$ is a functional inverse of Ackermann's function, and is very slowly growing (see [HS] for more detail concerning Ackermann's function; a brief review of the properties of this function is also given in Section 3). For $s>3$ the deep result of Szemeredi [Sz] states that $\lambda_{s}(n)=O\left(n \log ^{*} n\right)$, where ${ }^{*}$ the constant of proportionality depends on $s$, and where $\log ^{*} n$ is the smallest $i \geq 1$ such that $\epsilon_{i} \geq n$, where $\epsilon_{1}=2$ and $\epsilon_{l+1}=2^{\epsilon_{l}}$. (We use here the following standard notations. Let $f$, $g$ be two positive functions defined over the integers. Then $f(n)=O(g(n))$ (resp. $f(n)=\Omega(g(n)))$ if there exists a constant $C>0$ such that $f(n) \leq C g(n)$ (resp. $f(n) \geq C g(n))$ for all $n$. We write $f(n)=\Theta(g(n))$ if both $f(n)=O(g(n))$ and $f(n)=\Omega(g(n))$ hold, namely, if there cxist constants $C_{1}, C_{2}>0$ such that $C_{1} g(n) \leq f(n) \leq C_{2} g(n)$ for all $n$.)

In this paper wc derive the following upper bounds on $\lambda_{s}(n)$ for arbitrary $s>3$

\# In this paper all logarithms are with base 2. 


$$
\lambda_{s}(n) \leq n Q_{s}(\alpha(n))
$$

where $Q_{s}(\alpha)=O\left(\alpha^{O\left(\alpha^{s-3}\right)}\right)$ (a more precise definition of $Q_{s}$ is given in Section 5 below). These bounds improve Szemeredi's upper bounds [Sz]. We do not know however whether these bounds are tight (in fact we strongly suspect they are not). The proof of (1.1) involves a somewhat intricate inductive argument based on recurrence formulae which extend the formula used in [HS] for the case $s=3$. Another feature of the proof given here is that we analyze Davenport Schinzel sequences directly, and do not use any reduction of them to other combinatorial structures, as was done in [HS]. The results of this paper can also be modified to yield a more direct proof of the upper bound on $\lambda_{3}(n)$ given in [HS].

\section{Decomposition of Davenport-Schinzel sequences into chains}

Definition: Let $U$ be a $D S(n, s)$ sequence, and let $1 \leq t<s$. A $t$-chain $c$ is a contiguous subsequence of $U$ which is a Davenport-Schinzel secuence of order $t$.

Given $n, s, t$ and $U$ as above, we partition $U$ into disjoint $t$-chains, proceeding from left to right in the following inductive manner. Suppose that the initial portion $\left(u_{1}, \ldots, u_{j}\right)$ of $U$ has already been decomposed into $t$ chains. The next $t$-chain in our partitioning is then the largest subsequence of $U$ of the form $\left(u_{j+1}, \ldots, u_{k}\right)$ which is still a Davenport-Schinzel sequence of order $t$. We refer to this partitioning as the canonical decomposition of $U$ into $t$-chains, and let $m=m_{t}(U)$ denote the number of $t$-chains in this decomposition.

The problem of obtaining good upper bounds for the quantities

$$
\mu_{s, t}(n)=\max \left\{m_{t}(U): U \text { is a } D S(n, s) \text { sequence }\right\}
$$

seems quite hard for general $s$ and $t$. Nevertheless we have the following partial results. 
Proposition $2.1 \mu_{s, s-1}(n) \leq n$ and $\mu_{s, s-2}(n) \leq 2 n-1$.

Proof: Consider the first inequality. Let $U$ be a $D S(n, s)$ sequence, and let $\left(c_{1}, \ldots, c_{m}\right)$ be the canonical decomposition of $U$ into $(s-1)$-chains. Let $1 \leq j<m$, and suppose that $c_{j}=\left(u_{p}, u_{p}+1, \ldots, u_{q}\right)$. Let $b=u_{q+1}$. By our construction $c_{j}$ is a Davenport-Schinzel sequence of order $s-1$, whereas $c_{j} \|[b]$ is not. Hence there exists another symbol $a \neq b$ such that $c_{j}$ contains a subsequence of $s$ alternations of $a$ and $b$ ending at $a$. If $s$ is odd then this subsequence has the form

$$
\begin{array}{llllll}
a & b & a & \cdots & b & a
\end{array} \text {. }
$$

But then $c_{j}$ must contein both the first ocrurrence of $b$ and the last occurrence of $a$ in $U$, for otherwise $U$ would plainly contain one of the

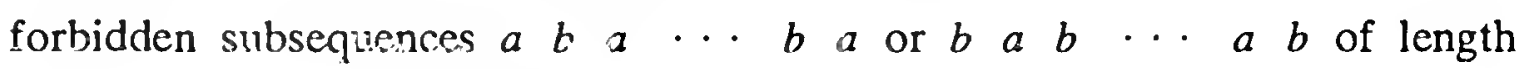
$s+2$. This implies that each chain $c_{j}$ must contain the last occurrence of some symbol (this obviously holds also for $j=m$ ), and hence we must have $m \leq n$. Similarly, if $s$ is even then $c_{j}$ must contain the subsequence

$$
\begin{array}{llllll}
b & a & b & \cdots & b & a
\end{array}
$$

of length $s$, and similar arguments to those used above imply that $c_{j}$ must contain both the first and the last occurrence of $a$, from which it follows once again that $m \leq n$.

To estimate $\mu_{s, s-2}(n)$ we proceed in much the same way. Specifically, suppose $s$ is odd, and consider an $(s-2)$-chain $c_{j}$ in the canonical decomposition of $U$, followed by a symbol $b$. Then there exists $a \neq b$ such that $c_{j}$ contains a subsequence of the form

$$
\begin{array}{llllll}
b & a & b & \cdots & b & a
\end{array}
$$


of length $s-1$. Now $c_{j}$ must contain either the first or the last occurrence of $a$ in $U$, for if $c_{j}$ contained neither of these occurrences, $U$ would have to contain a forbidden alternating subsequence of the form

$$
\begin{array}{lllllllll}
a & b & a & b & \cdots & b & a & b & a
\end{array}
$$

of length $s+2$. Since there are $n$ first occurrences and $n$ last occurrences of symbols in $U$, and since $c_{1}$ plainly contains at least two such first occurrences, it follows that $\mu_{s, s-2}(n) \leq 2 n-1$. Similar arguments can be used to handle the case in which $s$ is even. Q.E.D.

Definition: Let $n, m$ and $s$ be positive integers. We denote by $\psi_{s}(m, n)$ the maximal length of a $D S(n, s)$ sequense composed of at most $m$ 1-cheins.

In Section 4 we will cbtain upper bounds for $\psi_{s}(m, n)$. in order to apply these estimates to obtain upper bounds on $\lambda_{s}(n)$, we will need to estimate $\mu_{s, 1}(n)$, a task which we have tein unabic to handle successfully (for $s>3$ ). Instead, we will use the following approximation. Let $U$ be a $D S(n, s)$ sequence of maximal length, and let $\left(c_{1}, \ldots, c_{m}\right), m \leq n$, be its canonical decomposition into $(s-1)$-chains. We will replace each $(s-1)$-chain $c_{j}$ by a 1-chain $c_{j}^{*}$, by leaving in $c_{j}$ only one occurrence of each symbol appearing there. We will show in Section 5 that in this way we can obtain a $D S(n, s)$ sequence $U^{*}$ composed of at most $n$ chains, whose length is not much smaller than the length of $U$, i.e. than $\lambda_{s}(n)$. This will establish a connection between $\lambda_{s}(n)$ and $\psi_{s}(n, n)$, from which the desired upper bound on $\lambda_{s}(n)$ will be obtained. 


\section{Statement of Main Results}

In this section we state our main results, concerning the functions $\lambda$ and $\psi$. For this purpose, we briefly review first the definition and some basic properties of Ackermann's function and certain auxiliary functions ("generalized exponentials" - cf. [Ac]). A more detailed description of these functions can be found in [HS].

Let $N$ be the set of positive integers $1,2, \ldots$. Given a function $g$ from a set into itself, denote by $g^{(0)}$ the composition $g \circ g \circ \cdots \circ g$ of $g$ with itself $s$ times, for $s \in N$. Define inductively a sequence $\left\{A_{k}\right\}_{k=1}^{\infty}$ of functions from $\checkmark$ inio itself as follows:

$$
\begin{aligned}
& A_{1}(n)=2 n \\
& A_{k}(n)=A_{k}^{(n)}(1), \quad k \geq 2
\end{aligned}
$$

for all $n \in N$. Note that for all $k \geq 2$, the function $A_{k}$ satisfies

$$
\begin{aligned}
& A_{k}(1)=2, \\
& A_{k}(n)=A_{k-1}\left(A_{k}(n-1)\right), \quad n \geq 2 .
\end{aligned}
$$

In particular, $A_{2}(n)=2 A_{2}(n-1)$, thus $A_{2}$ is the "power function"

$$
A_{2}(n)=2^{n}, \quad n \in N .
$$

Then $A_{3}(n)=2^{A_{3}(n-1)}$, thus $A_{3}$ is the "tower function"

$$
A_{3}(n)=2^{2^{-^{2}}},
$$

with $n$ 2's in the exponential tower, for $n \in N$. Finally, put

$$
A(n)=A_{n}(n) .
$$

This is Ackermann's function (this is the variant used in [HS]). Ackermann's function $A$ grows very fast; its first values are: $A(1)=2, A(2)=4$, $A(3)=16$ and $A(4)$ is a tower of 655362 's. 
Given a strictly increasing fuction $g$ from $N$ into itself, its functional inverse is the function $\gamma$ from $N$ into itself given by

$$
\gamma(n)=\min \{s \geq 1: g(s) \geq n\} ;
$$

thus, $\gamma(n)=s$ if and only if $g(s-1)<n \leq g(s)$. In particular, let $\alpha_{k}$ and $\alpha$ denote the functional inverses of $A_{k}$ and $A$, respectively. Then, for all $n \in N$,

$$
\begin{gathered}
\alpha_{1}(n)=\left\lceil\frac{n}{2}\right\rceil, \\
\alpha_{2}(n)=\lceil\log n\rceil .
\end{gathered}
$$

The functions $\alpha_{k}$ are easily seen to satisfy the following recursive formula:

$$
\alpha_{k}(n)=\min \left\{s \geq 1: a_{k-1}^{(s)}(n):=1_{j} ;\right.
$$

that is, $\alpha_{k}(n)$ is the number of iterations of $\alpha_{k-1}$ needed to go from $n$ to 1 . In particular, $\alpha_{3}(n)$ is precisely $\log ^{*} n$, as defined in Section 1 .

All the functions $\alpha_{k}$ are non-decreasit.g, and converge to infinity with their argument. The same holds tor $\alpha$ too, which grows more slowly than any of the $\alpha_{k}$. Note that $\alpha(n) \leq 4$ for all $n \leq A(4)$ which is a tower with 65536 2's, thus $\alpha(n) \leq 4$ for all practical purposes.

The following property, which follows immediately from the above definitions, will be used in the sequel

$$
\alpha_{\alpha(n)}(n)=\alpha(n) .
$$

We will also need the following inequality, proved in [HS]:

$$
\alpha_{2 \alpha(n)-3}(n) \leq 3 .
$$

We can now state our results.

\section{MAIN THEOREM:}

$$
\lambda_{s}(n)=O\left(n \alpha(n)^{O\left(\alpha(n)^{s-3}\right)}\right)
$$


for $s \geq 3$ and $n \geq 1$.

It is easily verified that these bounds improve those of Szemeredi (which can be stated as $\left.\lambda_{s}(n) \leq C(s) n \alpha_{3}(n)\right)$, for each $s$ and sufficiently large $n$.

The Main Theorem is proven in Section 5. It is a consequence of the following Theorem, which yields upper bounds on the length of DavenportSchinzel sequences with a given number of 1-chains (i.e. on the function $\psi_{s}(m, n)$ defined in the preceding section).

\section{Theorem A:}

$$
\psi_{s}(m, n)=O\left((m+n) \alpha(m)^{O\left(\alpha(m)^{s-3}\right)}\right) .
$$

Theorem A will be proved in the following section.

\section{Upper bounds for $\psi_{s}(m, n)$}

In this section we establish almost linear upper bounds on the maximal length $\psi_{s}(m, n)$ of a $D S(n, s)$ sequence composed of at most $m$ 1-chains. Consider first the case $s=3$. This case has been analyzed in [HS], and the following upper bounds have been obtained (actually the bounds derived in [HS] were for another function, namely the maximal length of admissible path compression schemes on trees, but it can be easily verificd (cf. Proposition 4.1 of [HS]) that these same bounds also apply to our function $\left.\psi_{s}\right)$ :

$$
\psi_{3}(m, n) \leq 4 k m \alpha_{k}(m)+2 k n,
$$

for each $k \geq 1$. These bounds also yield, putting $k=2 \alpha(m)-3$ and making 
use of (3.2),

$$
\psi_{3}(m, n) \leq(24 m+4 n) \alpha(m) .
$$

Our aim is to establish inductively similar inequalities of the form

$$
\psi_{s}(m, n) \leq m F_{\omega}^{(s)}(\alpha(m))+n G_{\omega}^{(s)}(\alpha(m)),
$$

where $F_{\omega}^{(s)}, G_{\omega}^{(s)}$ are appropriate positive and monotonic functions, whose precise form will be specified below. Clearly, for the initial case $s=3$, (4.3) is obtained from (4.2) by choosing

$$
F_{\omega}^{(3)}(\alpha)=24 \alpha, \quad G_{\omega}^{(3)}(\alpha)=4 \alpha .
$$

Suppose next that (4.3) has been established for $s-1$, and consider the case of $s$. Our aim is to establish a recurrence formula for $\psi_{s}(m, n)$ which is a generalization of the formula obtained in Proposition 5.1 of [HS]. Specifically, we have

Proposition 4.1 Let $m, n \geq 1$, and let $b>1$ be a divisor of $m$. Then there exist integers $n^{*}, n_{1}, n_{2}, \ldots, n_{b} \geq 0$ such that

$$
n^{*}+\sum_{l=1}^{b} n_{l}=n
$$

and

$$
\begin{aligned}
\psi_{s}(m, n) \leq & \sum_{i=1}^{b} \psi_{s}\left(\frac{m}{b}, n_{l}\right)+2 \psi_{s}\left(b, n^{*}\right) \cdot G_{\omega}^{(s-1)}(\alpha(m))+ \\
& m\left(3+2 F_{\omega}^{(s-1)}(\alpha(m))+2 G_{\omega}^{(s-1)}(\alpha(m))\right) .
\end{aligned}
$$

Proof: Let $U$ be a $D S(n, s)$-sequence consisting of at most $m$ 1-chains $c_{1}, \ldots, c_{m}$ such that $|U|=\psi_{s}(m, n)$, and let $b>1$ be a divisor of $m$. Partition the sequence $U$ into $b$ layers $L_{1}, \ldots, L_{b}$ so that the layer $L_{l}$ consists of the $p=\frac{m}{b} 1$-chains $c_{(i-1) p+1}, c_{(l-1) p+2} \ldots, c_{i p}$. Call a symbol a internal 
to layer $L_{l}$ if all the occurrences of $a$ in $U$ are within $L_{l}$. A symbol will be called external if it is not internal to any layer. Suppose that there are $n_{i}$ internal symbols in layer $L_{l}$ and $n^{*}$ external symbols (thus $n^{*}+\sum_{l=1}^{b} n_{l}=n$ ).

To estimate the total number of occurrences in $U$ of symbols that are internal to $L_{l}$, we proceed as follows. Erase from $L_{l}$ all external symbols. Next scan $L_{t}$ from left to right and erase each' element which has become equal to the element immediately preceding it. This leaves us with a sequence $L_{i}^{*}$ which is clearly a $D S\left(n_{i}, s\right)$ sequence consisting of at most $\frac{m}{b} 1$-chains, and thus its length is at most $\psi_{s}\left(\frac{m}{i_{B}}, n_{i}\right)$. Moreover, if two equal internal elements in $L_{l}$ kave become adjacent after erasing the external symbols, then these two elements must have belonged to two distinct 1-chains, thus the total number of deletions of internal symbols is at most $\frac{m}{b}$.

Hence, summing over all layers, we conclude that the total contribution of internal symbols to $|U|$ is at most

$$
m+\sum_{i=1}^{b} \psi_{s}\left(\frac{m}{b}, n_{i}\right)
$$

Next, to estimate the contribution of external symbols to $|U|$, we argue as follows. For each $L_{i}$ consider separately external symbols whose first occurrence in $U$ appear to the left of $L_{i}$ (call them non-starting external symbols), and all the other external symbols which appear in $L_{i}$. Note that these latter symbols cannot have their rightmost occurrence within $L_{i}$, and for that reason we will refer to them as non-ending external symbols. Consider 
first non-starting symbols. To estimate their contribution to the length of $L_{l}$ we erase from $L_{l}$ all other symbols occuring there, and, if necessary, also erase each occurrence of a non-starting symbol which has become equal to the element immediately preceding it. As above, at most $\frac{m}{b}-1$ deletions of external non-starting symbols will be performed. Let $L_{i}^{*}$ be the resulting subsequence, and suppose that it is composed of $p_{l}$ distinct symbols.

We claim that $L_{l}^{*}$ is a $D S\left(p_{l}, s-1\right)$ sequence. Indeed, if this were not the case, $L_{l}^{*}$ would contain an alternating subsequence $W$ of two external nonstarting symbols $a, b$, which has length $s+1$ and which begins, say, with $a$. But since $b$ is assumed to be non-starting, there must exist at least one occurrence of $b$ to the left $0_{i}^{*} E_{i}$, which, culcatenated with $W$, yields a forbidden alternating subsequenze of length $s+2$ within $U$, contrary to assumptions.

Thus $L_{l}^{*}$ is a $D S\left(p_{l}, s-1\right)$ sequence, consisting of at most $\frac{m}{b} 1$-chains, so that, by induction hypothesis, its length is at most

$$
\psi_{s-1}\left(\frac{m}{b}, p_{l}\right) \leq \frac{m}{b} F_{\omega}^{(s-1)}\left(\alpha\left(\frac{m}{b}\right)\right)+p_{l} G_{\omega}^{(s-1)}\left(\alpha\left(\frac{m}{b}\right)\right) .
$$

Hence, summing over all layers, the total contribution of external nonstarting symbols is at most

$$
m-b+m F_{\omega}^{(s-1)}(\alpha(m))+\left(\sum_{l=1}^{b} p_{l}\right) G_{\omega}^{(s-1)}(\alpha(m)) .
$$

But if we leave, in each $L_{l}$, the leftmost occurrence of each external nonstarting symbol, and furthermorc delete (at most b) elements which have become equal to those inmediately preceding them, we obtain a $D S\left(n^{*}, s\right)$ 
sequence composed of at most $b$ chains, whose total length is at least $\sum_{i=1}^{b} p_{i}-b$. Thus

$$
\sum_{l=1}^{b} p_{l} \leq b+\psi_{s}\left(b, n^{*}\right) .
$$

Hence the total contribution of the external non-starting symbols to $|U|$ is at most

$$
m-b+m F_{\omega}^{(s-1)}(\alpha(m))+\left(b+\psi_{s}\left(b, n^{*}\right)\right) G_{\omega}^{(s-1)}(\alpha(m)) .
$$

Repeating the above argument once again to the analysis of the number of occurrences of external non-ending symbols in each of the layers $L_{l}$, we conclude that total contribution of these symbols to $|U|$ is also bounded by the bound just stated. Thus we obtain the following final inequality

$$
\begin{gathered}
\psi_{s}(m, n) \leq \sum_{l=1}^{b} \psi_{s}\left(\frac{m}{b}, n_{l}\right)+3 m-2 b+2 m F_{\omega}^{(s-1)}(\alpha(m))+ \\
2\left(b+\psi_{s}\left(b, n^{*}\right)\right) G_{\omega}^{(s-1)}(\alpha(m)) \leq \\
\sum_{l=1}^{b} \psi_{s}\left(\frac{m}{b}, n_{l}\right)+3 m+2 m\left(F_{\omega}^{(s-1)}(\alpha(m))+G_{\omega}^{(s-1)}(\alpha(m))\right)+ \\
2 \psi_{s}\left(b, n^{*}\right) G_{\omega}^{(s-1)}(\alpha(m)),
\end{gathered}
$$

as asserted. Q.E.D.

Proposition 4.2: Let $n, q \geq 1, k \geq 2$, and suppose that $m$ divides $A_{k}(q)$. Then

where

$$
\psi_{s}(m, n) \leq m q F_{k}^{(s)}\left(\alpha\left(A_{k}(q)\right)\right)+n G_{k}^{(s)}\left(\alpha\left(A_{k}(q)\right)\right),
$$

$$
F_{f}^{(s)}(\alpha)=0, \quad G_{f}^{(s)}(\alpha)=2
$$

and

$$
F_{k}^{(s)}(\alpha)=2 F_{k-1}^{(s)}(\alpha) \cdot G_{\omega}^{(s-1)}(\alpha)+3+2 F_{\omega}^{(s-1)}(\alpha)+2 G_{\omega}^{(s-1)}(\alpha)(4.7)
$$




$$
G_{k}^{(s)}(\alpha)=2 G_{k-1}^{(s)}(\alpha) \cdot G_{\omega}^{(s-1)}(\alpha),
$$

for $k \geq 2$.

Proof: We will use (4.5) repeatedly to obtain the series of upper bounds on $\psi_{s}$, stated in (4.6) for $k=2,3, \ldots$. At each step we choose $b$ in an appropriate manner, and estimate $\psi_{s}\left(b, n^{*}\right)$ using the bound obtained in the preceding step. This yields a recurrence relation on $\psi_{s}$ which we solve to obtain a better upper bound on $\psi_{s}$.

Specifically, we proceed by double induction on $k$ and $q$. To start this iterative process with $k=2$, suppose first that $m=A_{2}(q)=2^{q}$. Choose $b=2$ in (4.5); it is easily checked that $\psi_{s}\left(2, n^{*}\right)=2 n^{*}$ for all $n^{*}$, so that (4.5) yields

$$
\begin{gathered}
\psi_{s}(m, n) \leq m\left(3+2 F_{\omega}^{(s-1)}(\alpha(m))+? G_{\omega}^{(s-1)}(\alpha(m))\right)+ \\
4 n^{*} G_{\omega}^{(s-1)}(\alpha(m))+\psi_{s}\left(\frac{m}{2}, n_{1}\right)+\psi_{s}\left(\frac{m}{2}, n_{2}\right) .
\end{gathered}
$$

The solution to this recurrence relation, for $m$ a power of 2 and $n=n^{*}+n_{1}+n_{2}$ arbitrary, is

$\psi_{s}(m, n) \leq m \log m \cdot\left(3+2 F_{\omega}^{(s-1)}(\alpha(m))+2 G_{\omega}^{(s-1)}(\alpha(m))\right)+4 n G_{\omega}^{(s-1)}(\alpha(m))$.

(This is easily established by induction on $m$.)

To complete the argument for $k=2$, note that if $m$ divides $A_{2}(q)=2^{q}$ then $m$ is a power of 2 and $\log m \leq q$, thus

$$
\psi_{s}(m, n) \leq m q \cdot\left(3+2 F_{\omega}^{(s-1)}(\alpha(m))+2 G_{\omega}^{(s-1)}(\alpha(m))\right)+4 n G_{\omega}^{(s-1)}(\alpha(m)) .
$$

Thus if we put, as in (4.7),

$$
F_{2}^{(s)}(\alpha)=3+2 F_{\omega}^{(s-1)}(\alpha)+2 G_{\omega}^{(s-1)}(\alpha),
$$

and 


$$
G_{2}^{(s)}(\alpha)=4 G_{\omega}^{(s-1)}(\alpha),
$$

we can rewrite the preceding inequality as

$$
\psi_{s}(m, n) \leq m q F_{2}^{(s)}(\alpha(m))+n G_{2}^{(s)}(\alpha(m)),
$$

which, since $m \leq A_{2}(q)$, implies (4.6) for $k=2$.

In particular, we have for $m=1,2$ (and $q=1)$

$$
\psi_{s}(m, n) \leq m F_{2}^{(s)}(\alpha(m))+n G_{2}^{(s)}(\alpha(m)) \leq m F_{k}^{(s)}(\alpha(m))+n G_{k}^{(s)}(\alpha(m)),
$$

for all $k \geq 2$. Since $A_{k}(1)=2$ it follows that (4.6) holds for each $k \geq 2$ and $q=1$

Suppose next that $k>2$ and $q>1$, and that the induction hypothesis is true for all $k^{\prime}<k$ and $q^{\prime} \geq 1$, and for $k^{\prime}=k$ and all $q^{\prime}<q$. Observe that $A_{k}(q-1)$ is a divisor of $A_{k}(q)$ because they are both powers of 2 . Assume first that $m=A_{k}(q)$; let $t=A_{k}(q-1)$ and choose $b=\frac{m}{t}$, which is an integer dividing $m=A_{k}(q)=A_{k-1}(t)$. Hence by induction hypothesis (for $k-1$ and t) we have

$$
\begin{gathered}
\psi_{s}\left(b, n^{*}\right) \leq \frac{m}{t} t F_{k-1}^{(s)}\left(\alpha\left(A_{k-1}(t)\right)\right)+n^{*} G_{k-1}^{(s)}\left(\alpha\left(A_{k-1}(t)\right)\right)= \\
m F_{k-1}^{(s)}(\alpha(m))+n^{*} G_{k-1}^{(s)}(\alpha(m)) .
\end{gathered}
$$

Then (4.5) becomes

$$
\begin{aligned}
\psi_{s}(m, n) \leq & \sum_{i=1}^{b} \psi_{s}\left(t, n_{l}\right)+3 m+2 m\left(F_{\omega}^{(s-1)}(\alpha(m))+G_{\omega}^{(s-1)}(\alpha(m))\right)+ \\
& \left(2 m F_{k-1}^{(s)}(\alpha(m))+2 n^{*} G_{k-1}^{(s)}(\alpha(m))\right) \cdot G_{\omega}^{(s-1)}(\alpha(m)),
\end{aligned}
$$

Recall that we have defined

$$
\begin{gathered}
F_{k}^{(s)}(\alpha)=2 F_{k-1}^{(s)}(\alpha) \cdot G_{\omega}^{(s-1)}(\alpha)+3+2 F_{\omega}^{(s-1)}(\alpha)+2 G_{\omega}^{(s-1)}(\alpha), \\
G_{k}^{(s)}(\alpha)=2 G_{k-1}^{(s)}(\alpha) \cdot G_{\omega}^{(s-1)}(\alpha),
\end{gathered}
$$

Hence, using the induction hypothesis once more (for $k$ and $q-1$ ), we obtain 


$$
\begin{aligned}
\psi_{s}(m, n) \leq & \sum_{t=1}^{b}\left[t(q-1) F_{k}^{(s)}(\alpha(t))+n_{i} G_{k}^{(s)}(\alpha(t))\right]+ \\
& m F_{k}^{(s)}(\alpha(m))+n^{*} G_{k}^{(s)}(\alpha(m)) \leq \\
& m q_{k}^{(s)}(\alpha(m))+n G_{k}^{(s)}(\alpha(m))
\end{aligned}
$$

(because $n^{*}+\sum_{i=1}^{b} n_{i}=n$ ), as asserted.

Finally, assume $m$ divides $A_{k}(q)$, say $A_{k}(q)=p m$. Let $U$ be a $D S(n, s)$ sequence composed of at most $m$ 1-chains whose length is $\psi_{s}(m, n)$. Create $p$ copies of $U$, using $p$ disjoint sets of $n$ symbols each for these copies, and then concatenate all these copies to form a new sequence $U^{*} . U^{*}$ is obviously a $D S(p n, s)$ sequence composed of at most $p m=A_{k}(q)$ 1-chains, and its length is therefore

$$
p \psi_{s}(m, n) \leq \psi_{s}\left(A_{k}(q), p n\right) \leq q A_{k}(q) F_{k}^{(s)}\left(\alpha\left(A_{k}(q)\right)\right)+p n G_{k}^{(s)}\left(\alpha\left(A_{k}(q)\right)\right),
$$

which, divided by $p$, yields the required inequality. This completes the proof. Q.E.D.

Corollary 4.3: For all $m, n \geq 1$ and $k \geq 2$,

$$
\psi_{s}(m, n) \leq 2 m \alpha_{k}(m) F_{k}^{(s)}\left(\alpha^{\prime}\left(A_{k}\left(\alpha_{k}(m)\right)\right)\right)+n G_{k}^{(s)}\left(\alpha^{\prime}\left(A_{k}\left(\alpha_{k}(m)\right)\right)\right),
$$

where $\alpha_{k}$ is the functional inverse of $A_{k}$ as defined in Section 3.

Proof: Put $q=\alpha_{k}(m)$, so that $A_{k}(q-1)<m \leq A_{k}(q)$. Let $p=\left\lfloor\frac{A_{k}(q)}{m}\right\rfloor \geq 1$, then $A_{k}(q)<(p+1) m \leq 2 p m$. As in the preceding proof we have

$$
\begin{gathered}
p \psi_{s}(m, n) \leq \psi_{s}(p m, p n) \leq \psi_{s}\left(A_{k}(q), p n\right) \leq \\
q A_{k}(q) F_{k}^{(s)}\left(\alpha\left(A_{k}(q)\right)\right)+p n G_{k}^{(s)}\left(\alpha\left(A_{k}(q)\right)\right) \leq \\
2 p m q F_{k}^{(s)}\left(\alpha\left(A_{k}(q)\right)\right)+p n G_{k}^{(s)}\left(\alpha\left(A_{k}(q)\right)\right) .
\end{gathered}
$$

Dividing by $p$ we obtain the desired inequality. Q.E.D. 
Corollary 4.4: For all $m, n \geq 1$,

$$
\psi_{s}(m, n) \leq m F_{\omega}^{(s)}(\alpha(m))+n G_{\omega}^{(s)}(\alpha(m))
$$

where

$$
F_{\omega}^{(s)}(\alpha)=2 \alpha F_{\alpha}^{(s)}(\alpha), \quad G_{\omega}^{(s)}(\alpha)=G_{\alpha}^{(s)}(\alpha) .
$$

Proof: Put $k=\alpha(m)$. Then (3.1) implies that $\alpha_{k}(m)=\alpha(m)$, so that

$$
\alpha\left(A_{k}\left(\alpha_{k}(m)\right)\right)=\alpha(A(\alpha(m)))=\alpha(m) .
$$

Hence Corollary 3.1 implies that

$$
\psi_{s}(m, n) \leq 2 m \alpha(m) F_{\alpha(m)}^{(s)}(\alpha(m))+n G_{\alpha(m)}^{(s)}(\alpha(m)),
$$

as asserted. Q.E.D.

Proposition 4.2 and Coroliary 4.4 thus complete the inductive proof of (4.3) for all $s \geq 3$.

Proof of Theorem A: The inequalities (4.4) and (4.7) together yield a recurrence scheme for the functions $F_{\omega}^{(s)}$ and $G_{\omega}^{(s)}$. We can use these formulae to obtain more direct estimates on ihese functions as follows.

Proposition 4.5: For each $s>3$,

$$
\begin{aligned}
& G_{\omega}^{(s)}(\alpha) \leq 8^{\alpha^{s-3}} \alpha^{(\alpha-1)^{s-3}}, \\
& F_{\omega}^{(s)}(\alpha) \leq 11(2 \alpha)^{s-3} G_{\omega}^{(s)}(\alpha) .
\end{aligned}
$$

Proof: We will first establish (4.8a) using induction on $s$. For $s=3$ (4.8a) follows immediately from (4.4). Suppose $s>3$ and that (4.8a) has been established for $s-1$. It easily follows from (4.7) that

$$
G_{\omega}^{(s)}(\alpha) \leq 2\left[2 G_{\omega}^{(s-1)}(\alpha)\right]^{\alpha-1} .
$$

Thus by induction hypothesis we have

$$
G_{\omega}^{(s)}(\alpha) \leq 2\left[2 \cdot 8^{\alpha^{s-4}} \alpha^{(\alpha-1)^{s-4}}\right]^{\alpha-1}
$$




$$
\begin{gathered}
\leq 8^{\left(a^{s-4}+1\right)(\alpha-1)+1} \cdot \alpha^{(\alpha-1)^{s-3}} \\
\leq 8^{\alpha^{s-3}} \alpha^{(\alpha-1)^{s-3}},
\end{gathered}
$$

as asserted. This proves (4.8a) for all $s \geq 3$. Next consider (4.8b). It follows immediately from (4.4) that (4.8b) holds for $s=3$. Suppose $s>3$ and that (4.8b) has been established for $s-1$. Put

$$
a=2 G_{\omega}^{(s-1)}(\alpha), \quad b=3+2 F_{\omega}^{(s-1)}(\alpha)+2 G_{\omega}^{(s-1)}(\alpha) .
$$

Then (4.7) implies

$$
F_{k}^{(s)}(\alpha) \leq a F_{k-1}^{(s)}(\alpha)+b
$$

where $F_{f}^{(s)}(\alpha)=0$. This implies that

$$
F_{k}^{(s)}(\alpha) \leq \frac{a^{k-1}-1}{a-1} b
$$

Thus, putting $k=\alpha$, it follows that

$$
F_{\omega}^{(s)}(\alpha) \leq \frac{G_{\omega}^{(s)}(\alpha)-2}{2 G_{\omega}^{(s-1)}(\alpha)-1} \cdot\left(3+2 F_{\omega}^{(s-1)}(\alpha)+2 G_{\omega}^{(s-1)}(\alpha)\right) \cdot \alpha .
$$

To simplify the calculations, write $(4.8 \mathrm{~b})$ in the form

$$
F_{\omega}^{(s)}(\alpha) \leq D_{s}(\alpha) G_{\omega}^{(s)}(\alpha),
$$

Then the induction hypothesis implies that

$$
\begin{gathered}
F_{\omega}^{(s)}(\alpha) \leq \frac{\left[3+2\left(D_{s-1}(\alpha)+1\right) G_{\omega}^{(s-1)}(\alpha)\right] \cdot \alpha}{2 G_{\omega}^{(s-1)}(\alpha)-1} \cdot G_{\omega}^{(s)}(\alpha) \\
\leq\left(2 D_{s-1}(\alpha)+5\right) \alpha G_{\omega}^{(s)}(\alpha) .
\end{gathered}
$$

Hence (4.9) will continue to hold for $s$ too if we put

$$
D_{s}(\alpha)=\left(2 D_{s-1}(\alpha)+5\right) \alpha .
$$

The solution of this last recurrence formula is

$$
\begin{aligned}
D_{s}(\alpha) & =(2 \alpha)^{s-3} \cdot D_{3}(\alpha)+\frac{(2 \alpha)^{s-3}-1}{2 \alpha-1} \cdot 5 \alpha \\
& \leq(2 \alpha)^{s-3} \cdot D_{3}(\alpha)+2 \cdot(2 \alpha)^{s-4} \cdot 5 \alpha \\
= & (2 \alpha)^{s-3} \cdot\left(D_{3}(\alpha)+5\right)=11(2 \alpha)^{s-3}
\end{aligned}
$$


from which (4.8b) follows immediately. Q.E.D.

Theorem A now follows immediately from (4.3) and (4.8).

\section{Corollary 4.6:}

$$
\psi_{s}(n, n) \leq 12 n(2 \alpha(n))^{s-3} \cdot 8^{\alpha(n)^{s-3}} \cdot \alpha(n)^{(\alpha(n)-1)^{s-3}} .
$$

Proof: Immediatc.

\section{Upper bounds for $\lambda_{s}(n)$}

Having obtained the upper bounds on $\psi_{s}(m, n)$ in the preceding section, our next step is to use them to obtain almost linear upper bounds for $\lambda_{s}(n)$, by establishing a relationship between these two quantities. As already noted in Section 2 , this will be achieved by constructing a $D S(n, s)$ sequence $U^{*}$ whose length is sufficiently close to $\lambda_{s}(n)$, but which contains only at most $n$ 1-chains, and then by using $U^{*}$ to bound $\lambda_{s}(n)$ in terms of $\psi_{s}(n, n)$.

Details are as follows. We will prove, using induction on $s$, that for each $n \geq 1$

$$
\lambda_{s}(n) \leq n Q_{s}(\alpha(n))
$$

where $Q_{s}$ is a positive and monotonic function whose exact form will be specified below. The claim holds for $s=2$ if we put $Q_{2}(\alpha)=2$, as follows from the results of $[\mathrm{At}]$ and $[\mathrm{HS}]$.

Suppose the claim holds for all $2 \leq s^{\prime}<s$ and consider $\lambda_{s}(n)$. Let $U$ be a $D S(n, s)$ sequence of maximal length $\lambda_{s}(n)$, and let $\left(c_{1}, c_{2}, \ldots, c_{m}\right)$ be the canonical decomposition of $U$ into $(s-1)$-chains. By Proposition 2.1 we have $m \leq n$. For each $j=1, \ldots, m$ replace $c_{j}$ by the 1 -chain $c_{j}^{*}$ obtained by retaining in $c_{j}$ only the leftmost occurrence of each symbol appearing there. 
To obtain $U^{*}$ we first concatenate the 1 -chains $c_{1}^{*}, \ldots, c_{m}^{*}$ in order; then, proceeding from left to right, we erase every element that has become equal to the element immediately preceding it (at most $m \leq n$ elements will be erased). Suppose that $c_{j}$ contains $k_{j}$ distinct symbols, for $j=1, \ldots, m$. Since each $c_{j}$ is a $D S\left(k_{j}, s-1\right)$-sequence, we have by induction hypothesis

$$
\left|c_{j}\right| \leq \lambda_{s-1}\left(k_{j}\right) \leq k_{j} Q_{s-1}\left(\alpha\left(k_{j}\right)\right) \leq k_{j} Q_{s-1}(\alpha(n)) .
$$

Thus

$$
\left|c_{j}^{*}\right|=k_{j} \geq \frac{\left.\mid c_{j}\right\}}{Q_{s-1}(\alpha(n))}
$$

so that

$$
\left|U^{*}\right| \geq \sum_{j=1}^{m}\left|c_{j}^{*}\right|-n \geq \frac{\sum_{j=1}^{m}\left|c_{j}\right|}{Q_{s-i}(\alpha(n))}-n
$$

Hence

$$
\lambda_{s}(n)=|U|=\sum_{j=1}^{m}\left|c_{j}\right| \leqslant\left(n+\left|U^{*}\right|\right) \cdot Q_{s-1}(\alpha(n)) .
$$

But $\left|U^{*}\right|$ is plainly a $D S(n, s)$ sequence composed of at most $n$ 1-chains, so that by Theorem $\mathrm{A}$

$$
\left|U^{*}\right| \leq \psi_{s}(n, n) \leq n H_{s}(\alpha(n))
$$

where $H_{s}(\alpha(n))$ is as given in Corollary 4.6. We thus conclude that

$$
\lambda_{s}(n) \leq n\left(H_{s}(\alpha(n))+1\right) Q_{s-1}(\alpha(n)) .
$$

Hence (5.1) will also hold for $s$ if we put

$$
Q_{s}(\alpha)=\left(H_{s}(\alpha)+1\right) \cdot Q_{s-1}(\alpha)
$$

If we substitute the explicit form of $H_{s}$ into this recurrence formula and solve it, we obtain

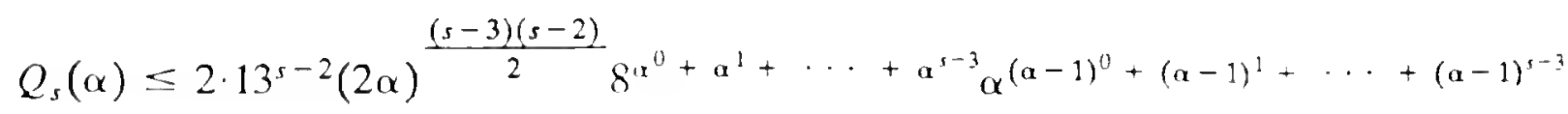


This completes the proof of the Main Theorem.

Remarks: (1) The bounds just obtained could be somewhat improved if we used the canonical decomposition of $U$ into $(s-2)$-chains rather than into $(s-1)$-chains. Nevertheless, the resulting bounds would be of the same asymptotic order of magnitıde.

(2) The case $s=3$ can also be handled by the above technique. In this case the chains $c_{j}$ are 2 chains, so that the length of each $c_{j}$ is at most $2 k_{j}-1$ (cf. [At], [HS]). The same reasoning as above would then imply

$$
\lambda_{3}(n) \leq 2\left(n+\psi_{3}(n, n)\right) .
$$

If we use this bound, and somewhat improve the analysis in Section 4 for the case $s=3$, we can obtain a more direct derivation of the bound $\lambda_{3}(n)=O(n \alpha(n))$ obtained in [HS].

(3) We do not have lower bounds for $\lambda_{s}(n)$ (other than those obtained in [HS] for $\lambda_{3}$ ), and we strongly suspect that the bounds (5.1) can be substantially improved. To do that, using the techniques developed in this paper, one would have (a) to improve the formula (4.5) by finding tighter estimates on the contribution of external symbols to the length of $U$, and (b) to establish a better connection between $\lambda_{s}$ and $\psi_{s}$, e.g. by establishing an explicit upper bound on $\mu_{s, 1}(n)$.

\section{Acknowledgement}

The author wishes to thank Sergiu Hart for several helpful discussions of the ideas presented in this paper. 


\section{REFERENCES}

[Ac] W. Ackermann, Zum Hilbertschen Aufbau der reellen Zahlen, Math. Ann. 99(1928), pp. 118-133.

[At] M. Atallah, Dynamic Computational Geometry, Proc. 24th Symp. on Foundations of Computer Science, 1983, pp. 92-99.

[DS] H. Davenport and A. Schinzel, A Combinatorial Problem Connected with Differential Equations, Amer. J. Math. 87(1965) pp. 684-694.

[Da] H. Davenport, A Combinatorial Problem Connected with Differential Equations, II, Acta Arithmetica 1'7(1971) pp. 363-372.

[HS] S. Hart and M. Sharir, Nonlinearity of Davenport-Schinzel Sequences and of Generalized Path Compression Schemes, Tech. Rept. 11/84, The Eskenasy Institute of Computer Sciences, Tel Aviv University, August 1984.

[RS] D.P. Roselle and R.G. Stanton, Some Properties of DavenportSchinzel Sequences, Acta Arithmetica 17(1971), pp. 355-362.

[Sz] E. Szemeredi, On a Problem by Davenport and Schinzel, Acta Arithmetica 25(1974) pp. 213-224.

[Ta] R.E. Tarjan, Efficiency of a Good but not Linear Set-union Algorithm, J. Assoc. Computing Machinery 22(1975), pp. 215-225. 
This book may be kcpt

\section{FOURTEEN DAYS}

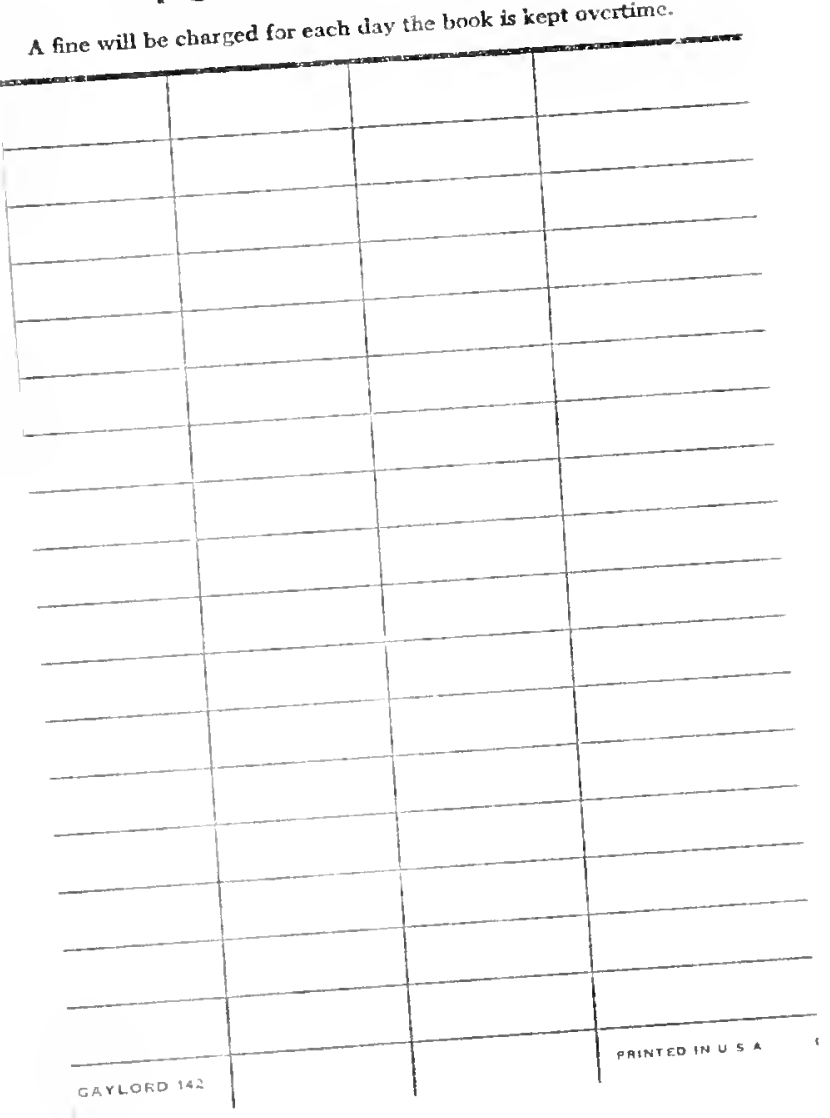

NYU COMPSCI TR-199

Sharir, Micha

Almost linear upper bounds on the length of general Davenport-Schinzel c.l

\section{NYU COMPSCI TR-199}

Sharir, Micha

Almost linear upper bounds on the length of general

Davenport-Schinzel c.l

\section{LIBRARY}

N.Y.U. Courant Institute of Mathematical Sciences 251 Mercer St. New York, N. Y. 10012 
